CARDIOVASCULAR MEDICINE

\title{
Local symptoms at the site of pacemaker implantation indicate latent systemic infection
}

\author{
D Klug, F Wallet, D Lacroix, C Marquié, C Kouakam, S Kacet, R Courcol
}

Heart 2004;90:882-886. doi: 10.1136/hrt.2003.010595

See end of article for authors' affiliations ......................

Correspondence to: Dr Didier Klug, Cardiology, CHRU Lille, Lille 59037, France; dklug@chru-lille.fr

Accepted 12 August 2003

\begin{abstract}
Background: To determine whether local complications at the site of pacemaker implantation indicate infection of the intravascular part of the lead as well as of the pacemaker pocket.

Methods: 105 patients admitted for local inflammatory findings, impending pacemaker or lead exteriorisation, frank pacemaker or lead exteriorisation, or overt infection were studied prospectively. After systematic lead extraction, the initial clinical presentation was related to the results of lead cultures. Results: Regardless of the initial presentation, the intravascular parts of the leads gave positive cultures in $79.3 \%$ of patients. Additionally, $91.6 \%$ of the cultures of the extravascular lead segments were positive, in contrast to $38.1 \%$ positivity for wound swab cultures. No clinical observations or laboratory investigations permitted identification of patients with negative lead cultures. In a subgroup of 50 patients with manifestations strictly limited to the pacemaker implantation site, cultures of intravascular lead segments were positive in $72 \%$. Infection recurred in $4 / 8$ patients without complete lead body extraction $(50 \%) \mathrm{v}$ $1 / 97$ patients $(1.0 \%)$ whose leads were totally extracted $(p<0.001)$.

Conclusions: Local complications at the site of pacemaker implantation are usually associated with infection of the intravascular part of the leads, with a risk of progressing to systemic infection. Such local symptoms should prompt the extraction of leads even in the absence of other infectious manifestations.
\end{abstract}

A vailable studies suggest that pacemaker lead related systemic infection is caused by progression of infection from the pacemaker pocket. ${ }^{1}$ If that concept is valid, local infection of the pocket should in many patients be associated with contamination of the leads and a subsequent risk of systemic infection. The poor prognosis of this complication would make it mandatory to remove the pacing system (leads included) in its entirety. As extraction of the leads is technically challenging and risky, and reimplantation of a new pacing system costly, ${ }^{2}$ precise indications for removal are highly desirable. We therefore studied 105 patients prospectively to test the policy of lead removal in cases where there were local symptoms at the site of implantation, and to validate the hypothesis that, whatever the initial presentation, positive lead cultures indicate systemic infection.

\section{METHODS}

Between 1995 and 2000, 105 consecutive patients (mean (SD) age, 70 (15) years) were included in this prospective study. The cohort mainly reflected secondary and tertiary care in an academic hospital covering an area of about four million people. Inclusion depended only on the cause for referral. The inclusion criterion was local symptoms at the pacemaker site. We excluded patients admitted with a diagnosis of septicaemia, septic shock, or overt infective endocarditis, with vegetation on echocardiography, or with a history of infective endocarditis. Patients with epicardial pacing systems were also excluded. Referrals generally occurred after several operative procedures at the pacemaker site, the mean number of all interventions including the implantation being 2.8 (1.8). The time interval between the last procedure at the pacemaker site and referral was 28.1 (28.0) months.

Inflammation, defined as local pain and redness of the skin, was present in eight patients; impending exteriorisation-manifested as adherence of the lead or pulse generator to the skin without cutaneous breakthrough-was observed in 16; and frank exteriorisation had occurred in 31. An infection with fistula, abscess, or purulent collection was present in the remaining 50 patients.

All patients underwent the following workup: complete clinical examination, three series of blood cultures, serial complete blood counts, creatinine and $\mathrm{C}$ reactive protein assays, and erythrocyte sedimentation rate (ESR). Imaging studies consisted of chest $x$ ray, transthoracic echocardiography (TTE), transoesophageal echocardiography (TOE), and ventilation/perfusion pulmonary scintigraphy, before any extraction attempt. The characteristics of patients are detailed in table 1 according to their initial presentation.

\section{Lead extraction}

All pacemakers and leads were systematically extracted except in five patients who refused lead extraction. Percutaneous extraction through the femoral vein was done as previously described. ${ }^{3}$ To prevent contamination of the lead by its passage through the pacemaker pocket, extraction from the femoral vein was undertaken by two operators, one liberating the proximal end of the lead and recovering the extravascular segment, the other recovering the intravascular segment of the lead from the femoral site.

\section{Blood and lead cultures}

Blood cultures were processed with an automated blood culture system (Bio Argos, Sanofi Diagnostics Pasteur, Marnes la Coquette, France).

Leads were cultured after extraction, and swab cultures from the pacemaker pocket were also obtained systematically. Bacteriological tests were done on the extravascular segment and two intravascular segments of the lead. These three parts were placed in sterile phosphate buffered saline and vortexed for one minute. An aliquot of the suspension was then plated on sheep blood agar under $\mathrm{O}_{2}$ and on chocolate agar under $\mathrm{CO}_{2}$. The plates were examined after 24 hours at $37^{\circ} \mathrm{C}$. In addition, a brain-heart infusion medium was inoculated with $0.1 \mathrm{ml}$ of the suspension for 15 days. An 
Table 1 Patient characteristics and results of cultures

\begin{tabular}{|c|c|c|c|c|c|}
\hline & $\begin{array}{l}\text { Inflammation } \\
(\mathrm{n}=8)\end{array}$ & $\begin{array}{l}\text { Impending ext } \\
\text { ( } n=16)\end{array}$ & $\begin{array}{l}\text { Exteriorisation } \\
(\mathrm{n}=31)\end{array}$ & $\begin{array}{l}\text { Local infection } \\
(\mathrm{n}=50)\end{array}$ & p Value \\
\hline Age (years) & $63(24)$ & $74(9)$ & $74(9)$ & $68(19)$ & NS \\
\hline $\operatorname{Sex}(F / M)$ & $1 / 7$ & $5 / 11$ & $5 / 26$ & $12 / 38$ & NS \\
\hline Procedures at pacemaker site (n) & $2.8(1.1)$ & $3.3(1.9)$ & $2.4(1.4)$ & $3.0(2.1)$ & NS \\
\hline $\begin{array}{l}\text { Time from last procedure to symptoms onset } \\
\text { (months) }\end{array}$ & $20(20)$ & $20(15)$ & $29(31)$ & $32(30)$ & NS \\
\hline Last procedure as initial implant & $1(12.5 \%)$ & $3(18.7 \%)$ & $9(29.0 \%)$ & $12(24.0 \%)$ & NS \\
\hline History of local complications & $3(37.5 \%)$ & $8(50.0 \%)$ & $6(19.3 \%)$ & $11(22.0 \%)$ & NS \\
\hline Intermittent fever & $1(12.5 \%)$ & $2(26.3 \%)$ & $4(12.9 \%)$ & $8(16.0 \%)$ & NS \\
\hline Clinical pulmonary manifestations & 0 & $1(6.2 \%)$ & $1(3.2 \%)$ & $4(8.0 \%)$ & NS \\
\hline Raised WBC count & $2(25.0 \%)$ & $1(6.2 \%)$ & $5(16.1 \%)$ & $7(14.0 \%)$ & NS \\
\hline Laboratory findings of inflammation & $2(25 \%)$ & $5(31.2 \%)$ & $12(38.7 \%)$ & $20(40.0 \%)$ & NS \\
\hline Pulmonary embolism & $3(37.5 \%)$ & $1(6.2 \%)$ & $3(9.7 \%)$ & $10(20.0 \%)$ & NS \\
\hline Positive blood culture (by Duke criteria) & $1(12.5 \%)$ & $1(6.2 \%)$ & $1(3.2 \%)$ & $2(4 \%)$ & NS \\
\hline Lead extraction refused or failed & 3 & 3 & $2^{10.21}$ & 2 & NS \\
\hline Wound swab culture & $3(37.5 \%)$ & $8(50.0 \%)$ & $11(35.5 \%)$ & $18(36.0 \%)$ & NS \\
\hline Positive extravascular lead cultures & $8 / 8(100 \%)$ & $13 / 14(92.8 \%)$ & $25 / 26(96.1 \%)$ & $41 / 47(87.2 \%)$ & NS \\
\hline Positive intravascular lead cultures & $5 / 6(83.3 \%)$ & $10 / 13(76.9 \%)$ & $22 / 27(77.7 \%)$ & $36 / 46(78.3 \%)$ & NS \\
\hline$S$ epidermidis & $5(100 \%)$ & $8(80.0 \%)$ & $16(76.1 \%)$ & $22(61.1 \%)$ & NS \\
\hline Other coagulase negative staphylococcus & $1(20 \%)$ & $2(20.0 \%)$ & $4(19.0 \%)$ & $10(27.8 \%)$ & NS \\
\hline$S$ aureus or other microorganism & 0 & $1(10.0 \%)$ & $6(28.6 \%)$ & $13(36.1 \%)$ & NS \\
\hline$>1$ microorganism & $2(40 \%)$ & $3(30.0 \%)$ & $2(9.5 \%)$ & $5(13.9 \%)$ & NS \\
\hline
\end{tabular}

automated system (Api-System-Expression, BioMérieux, Marcy l'Etoile, France) was used for identification of each colony species.

According to the Duke criteria, ${ }^{4}$ infective endocarditis was considered present when the culture of an intravascular part of a lead was positive.

\section{Validation of lead cultures}

The reliability of lead cultures after percutaneous extraction was evaluated by placing 14 unused sterile leads upon the operating table during 14 procedures for infected lead extraction. The leads were similarly cut into three segments at the end of the procedure and sent to the microbiology laboratory without any further information.

A control group of 11 patients was formed, in whom lead dysfunction required removal and replacement in the absence of any local symptom.

\section{Medical treatment}

Only 14 patients had received antibiotics before their referral for lead extraction (methicillin in 12, vancomycin in one, and pristinamycin in one). The antibiotic had been systematically withdrawn at the time of admission, and the extraction procedure was done without antimicrobial cover. All patients received intravenous antibiotics after the extraction procedure. These were chosen according to the microorganism isolated in blood cultures. If these cultures were negative, antistaphylococcal treatment was given empirically. The drug regimens were modified according to the microorganisms identified in the lead cultures. Treatment was given intravenously for two weeks after lead extraction, then orally for four weeks.

In pacing dependent patients, an epicardial pacing system was inserted before extraction of the endocardial system. Otherwise, the indication for permanent pacing was reevaluated and, if indicated, a new transvenous permanent pacing system was implanted 15-60 days later.

\section{Follow up}

Patients were examined at the outpatient clinic 12 weeks after discharge and yearly thereafter. Additional follow up information was obtained by telephone contacts with patients and their primary physicians.

\section{Statistical analysis}

Measurements between groups were compared by analysis of variance (ANOVA) followed by the appropriate post test or by Student's $t$ test according to the case. Comparisons were two tailed and statistical analyses were done using the Prism software package (GraphPad, USA). A probability value of $\mathrm{p}<0.05$ was considered significant.

\section{RESULTS}

\section{Blood cultures}

A community acquired Staphylococcus aureus species was isolated from two separate blood cultures in two patients. Persistently positive blood cultures with a coagulase negative staphylococcus, consistent with endocarditis according to the Duke criteria, ${ }^{4}$ were found in one of eight patients with inflammation, one of 16 with impending exteriorisation, one of 31 with frank exteriorisation, and none of 50 with local infection (NS). A single positive blood culture identifying a coagulase negative staphylococcus was obtained in one of eight, one of 16, four of 31, and three of 50 patients with inflammation, impending exteriorisation, frank exteriorisation, and local infection, respectively (NS). Blood cultures were negative in the remaining 91 patients.

\section{Lead cultures}

Lead culture results are summarised in tables 1 and 2. In seven patients who underwent prolonged and technically complicated procedures, lead cultures were considered unreliable and were discarded from the analysis. Additionally, data pertaining to the intravascular segment of the lead were not available in the five patients mentioned above who refused extraction, and in one patient in whom we were unable to remove any segment of the intravascular part of the leads. Overall, cultures of the whole leads or of a segment of their intravascular parts were obtained in 92 patients. Cultures of these segments were positive in 73 of these (79.3\%). Coagulase negative staphylococcal species, and $S$ epidermidis specifically, were isolated from $73.9 \%$ and $55.4 \%$ of these segments, respectively. The characteristics of patients with and without intravascular lead infections are compared in table 2. Fever, pulmonary embolism, increase white cell count, increased $\mathrm{C}$ reactive protein, and positive blood cultures predicted positive cultures from the intravascular 
Table 2 Comparison of patients with positive $v$ negative intravascular lead cultures

\begin{tabular}{llll}
\hline Number of patients & $\begin{array}{l}\text { Positive } \\
(\mathbf{n = 7 3 )}\end{array}$ & $\begin{array}{l}\text { Negative } \\
(\mathbf{n}=19)\end{array}$ & p Value \\
\hline Age (years) & $70.0(1.7)$ & $70.4(3.4)$ & NS \\
Sex (F/M) & $13 / 60$ & $5 / 17$ & NS \\
Immunological deficiency & 16 & 6 & NS \\
Number of procedures & $2.9(0.2)$ & $2.3(0.2)$ & 0.002 \\
Number of leads & $2.1(0.1)$ & $1.5(0.1)$ & 0.001 \\
Time from last procedure to presentation (months) & $27.8(2.6)$ & $36.2(8.8)$ & NS \\
History of previous local complications & 28 & 4 & NS \\
Local findings (inflammation, IExt, Ext, infection) & $5 / 10 / 22 / 36$ & $1 / 3 / 5 / 10$ & NS \\
Fever & 12 & 1 & NS \\
Clinical pulmonary manifestations & 6 & 1 & NS \\
Pulmonary embolism & 15 & 0 & 0.03 \\
Increased WBC count & 12 & 0 & NS \\
Increased C reactive protein & 33 & 5 & NS \\
Positive blood culture & 5 & 0 & NS \\
Isolated local pathological findings & 31 & 12 & NS \\
\hline Values are mean (SD) or $n$. & & & \\
Ext, exteriorisation; F, female; IExt, impending exteriorisation; & M, male; WBC, white blood cells. & \\
\hline
\end{tabular}

part of the leads with a very high specificity $(0.95,1,1,0.74$, and 1 , respectively), but with a very low sensitivity $(0.16$, $0.20,0.17,0.45$, and 0.07 , respectively).

Each of the five species of microorganism that had been isolated from at least two separate blood cultures was always found on the intravascular lead culture. Additionally, when the culture of an intravascular lead segment was positive, the same microorganism was found on the culture of the extravascular portion of the lead. There were no patients with a positive culture on the proximal intravascular segment and a negative culture on the distal intravascular segment. Cultures of the extravascular lead segment were available in 95 patients and were positive in 87 of these (91.6\%). In contrast, wound swab cultures were positive in only $38.1 \%$ of patients.

\section{Validation of lead cultures}

All 14 control leads tested in the operating room were free of infection related findings on direct examination, and a single culture of their 42 segments was positive for $S$ epidermidis.

Cultures from the 11 leads extracted for dysfunction in control patients were all negative.

In eight patients of our population, restriction endonuclease analysis of DNA of the staphylococcus cultured from the different parts of the leads confirmed the same monoclonal origin of the microorganism found simultaneously on the extravascular and the intravascular segments.

\section{Significance of isolated local symptoms}

After detailed investigations, a subgroup of 50 patients was defined as follows: clinical findings strictly limited to the pacemaker implantation site; fever, laboratory markers of inflammation, and blood cultures negative; and pulmonary and cardiac imaging (TOE included) normal. In 36 of these $(72 \%)$ the cultures of the intravascular and extravascular parts of the leads were positive. In nine additional patients, only the extravascular part of the lead gave a positive culture.

\section{Outcomes}

There were two procedural deaths (1.9\%). These deaths occurred more than 20 minutes after completion of lead extraction; the patients collapsed suddenly and died in septic shock. Normal necropsy examinations confirmed this diagnosis. Thus these procedural deaths were not a result of mechanical problems but were directly related to the infective process.
Duration of follow up was 24 (17) months. Six patients $(5.7 \%)$ died after discharge, one presumably from septic shock after having declined lead removal. The five remaining deaths were unrelated to pacemaker infection. Infection recurred in five patients $(4.7 \%)$. In four of these, recurrence occurred with systemic symptoms including one case of septic shock, and triscupid endocarditis in another case. Overall, full removal of pacing leads was not achieved or not attempted (consent not given) in eight patients, among whom four recurrences of infection $(50.0 \%)$ were observed. In contrast, a single recurrence was observed among the 97 patients whose leads were fully removed (1.0\%) $(\mathrm{p}<0.0001)$.

\section{DISCUSSION}

The main result of our study is that local symptoms at the site of pacemaker implantation are highly correlated (79.3\%) with a positive culture of the intravascular part of the leads. This strong and striking correlation is present whatever the nature of these symptoms, and even in cases where the symptoms were an isolated finding after an extensive work up for infective endocarditis. Lead removal is associated with a favourable outcome; the converse was true when this removal was not achieved.

Our results confirm the mechanism of intravascular infection originating from the pacemaker pocket with subsequent risk of progressing to systemic infection or right heart endocarditis. Da Costa and colleagues have shown that pacing system infections are mainly caused by local contamination by local bacteriological flora during implantation. ${ }^{1}$ Skin microorganisms migrate from the insertion site along the surface of the lead, colonising its distal intravascular part and ultimately infecting the blood stream. Studies by transmission and scanning electron microscopy have shown that nearly all indwelling vascular catheters are colonised by microorganisms. ${ }^{5}$

There is agreement that all the implantable material should be extracted in patients with septicaemia related to an infected pacing system. ${ }^{6-21}$ On the other hand, there is no clear consensus with respect to the management of pathological findings limited to the pacemaker implantation site, though the trend is toward extraction of the entire system when the cause is infectious, rather than conservative management for purely mechanical complications. ${ }^{22}$ Patients with class I indications for lead removal according to the NASPE policy ${ }^{23}$ were excluded from our study, as clearly stated in the Methods section. Our study dealt with class II 
indications in order to confirm or invalidate the policy of hardware removal. The study highlights the difficulty of excluding the presence of an infection on the grounds of clinical observation or detailed investigations. Griffith and colleagues have proposed a two step approach primarily based on the results of wound swab cultures, reporting the need for a new contralateral pacemaker in fewer than $40 \%$ of patients after a mean follow up of 21.3 months. ${ }^{22}$ However, the lack of reliability of their wound swab cultures was illustrated by negative results in nearly $30 \%$ of patients who ultimately developed recurrences. ${ }^{22}$ In our study, $38.1 \%$ of wound swab cultures were positive, contrasting with $91.6 \%$ of positive lead cultures; one may question the appropriateness of exposing patients to an approach based on a test with such a low sensitivity.

In this series, some patients without complete lead removal had recurrences with systemic infection not present at the time of recruitment. As failure rates of percutaneous lead extraction procedures increase with the age of the lead, ${ }^{23}$ postponement of the procedure may not only allow the growth of large vegetations, with a risk of massive pulmonary embolism at the time of extraction, but also renders extraction more difficult. On the other hand, lead extraction procedures are technically challenging and carry a risk of death. In this study, though procedural mortality was $1.9 \%$, this risk has to be compared with the risk of death in cases of uncontrolled infection-infection recurred in half the patients without complete extraction of the lead body, and this recurrence was fatal in one of these patients $(12.5 \%)$.

Our studies were not designed to provide recommendations about antibiotic treatment. However, staphylococci adherent to polymer surfaces and their capacity for biofilm formation contribute to the pathogenesis of infections with implanted medical devices. ${ }^{24}$ The sterilisation of infected implanted devices by antibiotics is extremely difficult. This is why prolonged use of intravenous antibiotics is not the recommended form of treatment for infected pacemakers if the implanted material has not been removed. ${ }^{15}{ }^{23}$ The very small proportion of patients with antibiotic treatment before referral in this series could explain the $38.1 \%$ of positive wound swab cultures in our series in comparison with the $22.5 \%$ in the series by Griffith and colleagues ( $p=0.04)$. However, antibiotic treatment before lead extraction would probably not modify the results of cultures obtained directly from the implanted material, especially lead cultures. Several investigators have proposed conservative treatment, ${ }^{25-29}$ and conservative management is required if lead extraction is impossible, unsuccessful, judged too risky, or refused. In cases where infected leads remain in place, prolonged follow up is required and perhaps prolonged antibiotic treatment.

Almost all patients were referred from secondary care centres, and then admitted to our tertiary centre, specialising in pacemaker lead extractions. It should be emphasised that 28 patients (table 1, history of local complication) had undergone several previous therapeutic attempts because of their local symptoms before referral and inclusion in this study. This may have introduced a referral bias, with a selection of a larger proportion of infectious complications resistant to the previous conservative management usually undertaken in the referral centres. This bias, however, seems to have had a limited impact because the nature of the last procedure preceding inclusion in the study, and the percentage of patients with previous conservative surgery for local complications, were not statistically different in those with and without positive lead cultures.

\section{Conclusions}

Regardless of the initial clinical presentation, complications at the pacemaker implantation site are associated with an infectious process in the vast majority of cases. Furthermore, even in the absence of signs or symptoms of systemic infection, the process is rarely limited to the extravascular components of the pacing system. Consequently, the management of local symptoms-even when apparently confined to the pulse generator implantation site-should prompt the extraction of the entire system. A recurrence rate of infection in $50 \%$ of patients in whom the leads were not fully removed strongly supports this recommendation.

\section{Authors' affiliations}

D Klug, D Lacroix, C Marquié, C Kouakam, S Kacet, Department of Cardiology, University of Lille, Lille, France

F Wallet, R Courcol, Department of Bacteriology, University of Lille

\section{REFERENCES}

1 Da Costa A, Leliévre H, Kirkorian G, et al. Role of preaxillary flora in pacemaker infections A prospective study. Circulation 1998:97:1791-5.

2 Smith HJ, Fearnot NE, Byrd CL, et al. Five-years experience with intravascular lead extraction. U.S. Lead Extraction Database. Pacing Clin Electrophysiol 1994;17:2016-20.

3 Jarwé $M$, Klug D, Beregi JP, et al. Single center experience with femoral extraction of permanent endocardial pacing leads. Pacing Clin Electrophysiol 1999;22:1202-9.

4 Bayer AS, Ward Jl, Ginzton LE, et al. Evaluation of new clinical criteria for the diagnosis of infective endocarditis. Am J Med 1994;96:211-19.

5 Raad I. Intravascular-catheter-related infections. Lancet 1998;351:893-8.

6 Conklin EF, Giannelli S, Nealon TF. Four hundred consecutive patients with permanent transvenous pacemakers. J Thorac Cardiovasc Surg 1975;69:1-7.

7 Bluhm G. Pacemaker infections. A clinical study with special reference to prophylactic use of some isoxazolyl penicillins. Acta Med Scand 1985;699(suppl): 1-62.

8 Bloch K, Russi E. Right heart endocarditis. Schweiz Med Wochenschr 1989;119:1664-72.

9 Glock Y, Sabatier J, Salvador-Mazencq M, et al. Endocarditis on cardiac pacemaker endocavitary electrodes. A propos of 7 cases. Arch Mal Coeur Vaiss 1986:79:483-8.

10 Loffler S, Kasper J, Postulka J, et al. Septic complications in patients with permanent pacemakers. Cor Vasa 1988;30:400-4.

11 Morgan G, Ginks W, Siddons H, et al. Septicemia in patients with an endocardial pacemaker. Am J Cardiol 1979;44:221-4.

12 Rubio-Alvarez J, Duran-Munoz D, Sierra-Quiroga J, et al. Right heart endocarditis and endocardial pacemakers. Ann Thorac Surg 1989;48: 147-51

13 Choo MH, Holmes DR, Gersh BJ, et al. Permanent pacemaker infections. Characterization and management. Am J Cardiol 1981;48:559-64.

14 Harcombe AA, Newell SA, Ludman PF, et al. Late complications following permanent pacemaker implantation or elective unit replacement. Heart 1998:80:240-4.

15 Chua JD, Wilkoff BL, Lee I, et al. Diagnosis and management of infections involving implantable electrophysiologic cardiac devices. Ann Intern Med 2000; 133:604-8

16 Lewis $A B$, Hayes DL, Holmes DR, et al. Update on infections involving permanent pacemakers. Characterization and management. J Thorac Cardiovasc Surg 1985;89:758-63.

17 Hajrula A, Jarvinen A, Virtanen KS, et al. Pacemaker infections: treatment with total or partial pacemaker system removal. Thorac Cardiovasc Surg 1985;33:218-20.

18 Klug D, Lacroix D, Savoye C, et al. Systemic infection related to endocarditis on pacemaker leads: clinical presentation and management. Circulation 1997;95:2098-107.

19 Molina JE. Undertreatment and overtreatment of patients with infected antiarrhythmic implantable devices. Ann Thorac Surg 1997;63:504-9.

20 Victor F, De Place C, Camus C, et al. Pacemaker lead infection: echocardiographic features, management, and outcome. Heart 1999:81:82-7.

21 Camus $C$, Leport $C$, Raffi $F$, et al. Sustained bacteremia in 26 patients with a permanent endocardial pacemaker: assessment of wire removal. Clin Infect Dis 1993; 17:46-55.

22 Griffith MJ, Mounsey JP, Bexton RS, et al. Mechanical, but not infective, pacemaker erosion may be successfully managed by re-implantation of pacemakers. Br Heart J 1994;71:202-5.

23 Love CJ, Wilkoff BL, Byrd CL, et al. Recommendations for extraction of chronically implanted transvenous pacing and defibrillator leads: indications, facilities, training. North American Society of Pacing and Electrophysiology Lead Extraction Conference Faculty. Pacing Clin Electrophysiol 2000;23:544-51

24 Klug D, Wallet F, Kacet $S$, et al. Involvement of adherence and adhesion Staphylococcus epidermidis genes in pacemaker lead-associated infections. $J$ Clin Microbiol 2003;41:3348-50.

25 Jara FM, Toledo-Pereyra L, Lewis JW, et al. The infected pacemaker pocket J Thorac Cardiovasc Surg 1979;78:298-300. 
26 Har-Shai $Y$, Amikam S, Bolous $M$, et al. The management of soft tissue complications related to pacemaker implantations. J Cardiovasc Surg 1994;35:211-17.

27 Lee JH, Geha AS, Rattehalli NM, et al. Salvage of infected ICDs: management without removal. Pacing Clin Electrophysiol 1996;19:437-42.
28 Baddour LM. Long-term suppressive antimicrobial therapy for intravascular device-related infections. Am J Med Sci 2001;322:209-12.

29 Yamada M, Takeuchi S, Shiojiri Y, et al. Surgical lead-preserving procedures for pacemaker pocket infection. Ann Thorac Surg 2002;74:1494-9; discussion 1499.

\section{IMAGES IN CARDIOLOGY}

Electrocardiographic gated multislice computed tomography of Uhl's anomaly

U hl's anomaly of the right ventricle is an unusual cardiac disorder with almost complete absence of right ventricular myocardium, normal tricuspid valve, and preserved septal and left ventricular myocardium. A 12 year old boy was admitted with the complaint of fatigue with effort. A holosystolic murmur was heard on left lower sternal border. Electrocardiography showed right atrial dilation and low voltage in all leads. Cardiomegaly was apparent on chest $x$ ray. Echocardiography revealed a greatly enlarged right atrium and ventricle. The right ventricular free wall seemed very thin.

Electrocardiographic gated multislice computed tomography (CT) showed an extremely thin walled right ventricle with almost complete absence of right ventricular free wall myocardium (panels A and B below). The interventricular septum and left ventricular myocardium were normal. Although magnetic resonance imaging can also show the absence of right ventricular myocardium, three dimensional reconstructed multislice CT imaging (right panel) makes it easier to see the difference between the normal left ventricular myocardium and the thin walled right ventricle.

This is the first case of Uhl's anomaly that has been demonstrated by multislice CT, and the images suggest that multislice CT can be used for definition of many cardiac pathologies.

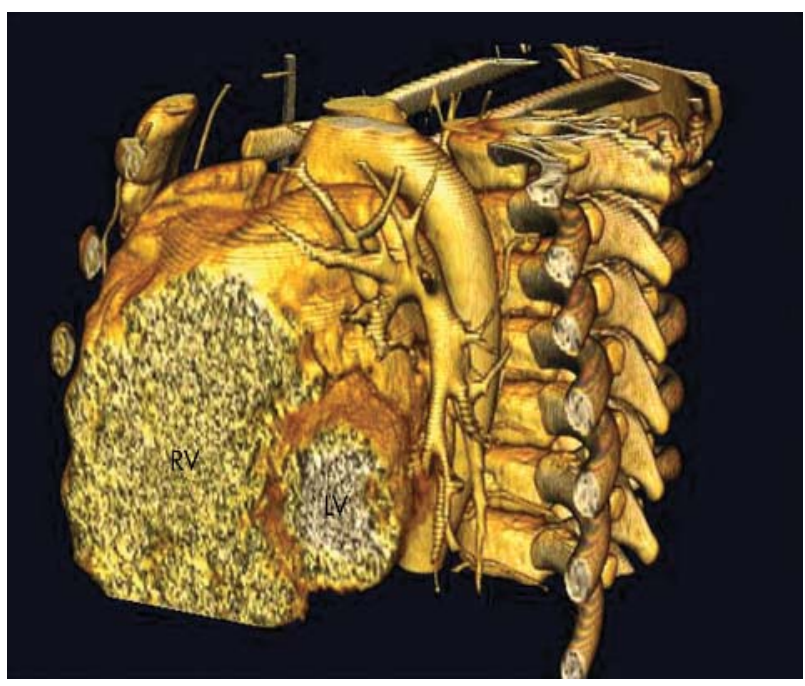

Three dimensional reconstructed CT image makes it easier to see the absence of right ventricular free wall myocardium.
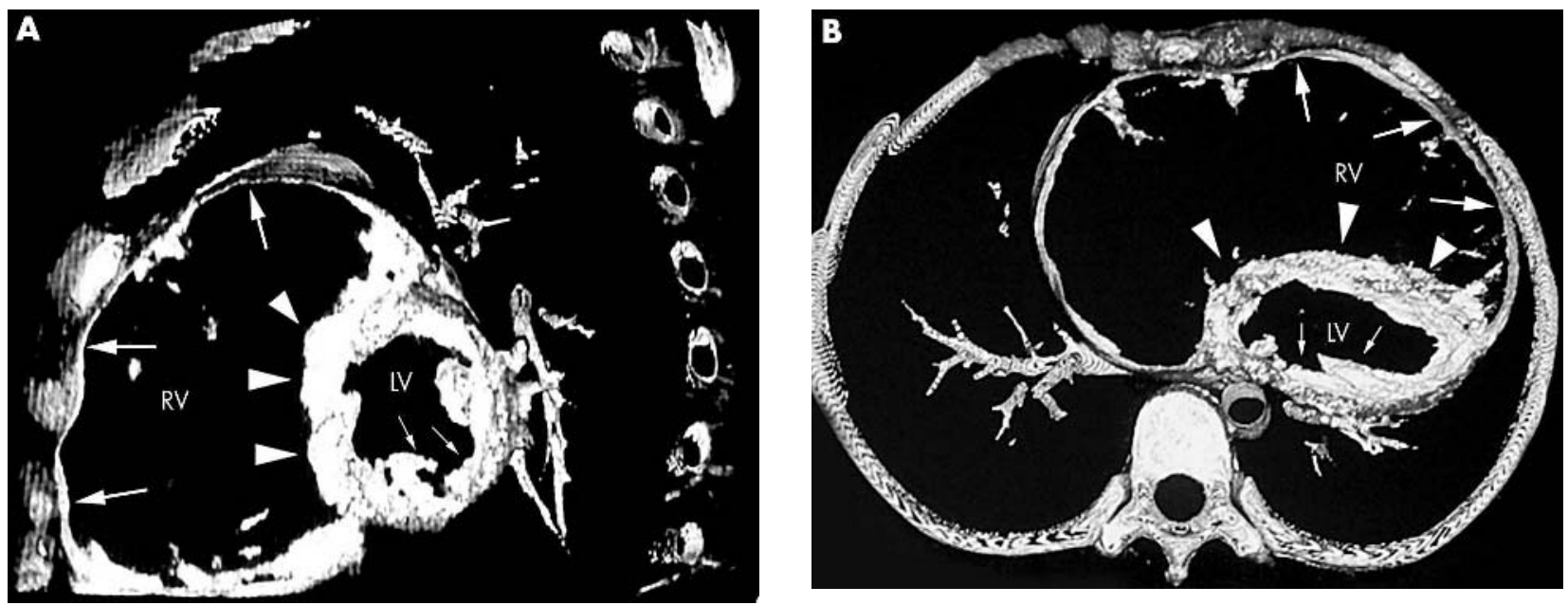

Reconstructed multislice CT images in sagittal (A) and axial (B) views show the extremely thin walled right ventricle (thick arrows), normal interventricular septum (arrowheads) and left ventricular myocardium (thin arrows). 